\title{
Microbiology Specimen Reference Identifier
}

National Cancer Institute

\section{Source}

National Cancer Institute. Microbiology Specimen Reference Identifier. NCI Thesaurus.

Code C87914.

A character or string used to name, or characterize a microbiology specimen reference. 